\title{
Association of myeloperoxidase, homocysteine and high-sensitivity C-reactive protein with the severity of coronary artery disease and their diagnostic and prognostic value
}

\author{
MINJU CHENG ${ }^{1}$, MINJING CHENG $^{2}$ and QINGMIN WEI ${ }^{1}$ \\ ${ }^{1}$ Department of Cardiology, Xingtai People's Hospital, Xingtai, Hebei 054000; ${ }^{2}$ Department of Cardiology, \\ The Third Hospital of Hebei Medical University, Shijiazhuang, Hebei 050051, P.R. China
}

Received May 17, 2019; Accepted January 6, 2020

DOI: $10.3892 / \mathrm{etm} .2020 .8817$

\begin{abstract}
In the present study, the association between the severity of coronary artery disease (CAD) and myeloperoxidase (MPO), homocysteine (Hcy) and high-sensitivity C-reactive protein (hs-CRP) was assessed and their diagnostic and prognostic value was determined. A total of 112 patients with CAD [patient group (PG)] and 112 healthy participants who visited the hospital for physical examinations [control group (CG)] were enrolled in the present study. The plasma levels of MPO, Hcy and hs-CRP were compared between the two groups. According to the arteriography results, the patients were further divided into the single-vessel disease group (SVG), double-vessel disease group (DVG) and multi-vessel disease group (MVG). The Gensini scores of the three groups were evaluated according to the Gensini score standard. The correlations between the expression of MPO, Hcy or hs-CRP and the Gensini score of the PG were analyzed. The patients' major adverse cardiovascular event (MACEs) were recorded over 6 months and compared, and the predictive values of MPO, Hcy and hs-CRP regarding MACEs were determined by receiver operating characteristics analysis. The results indicated that the levels of MPO, Hcy and hs-CRP in the $\mathrm{PG}$ were higher than those in the $\mathrm{CG}(\mathrm{P}<0.05)$. The Gensini score and the expression of MPO, Hcy and hs-CRP in the MVG were higher than those in the SVG and the DVG, and the Gensini score and the expression of MPO, Hcy and hs-CRP in the DVG were higher than those in the SVG $(\mathrm{P}<0.05)$. There was a positive correlation between the Gensini score and the expression of MPO $(r=0.814, \mathrm{P}<0.05)$, Hcy $(r=0.774, \mathrm{P}<0.05)$ and hs-CRP $(r=0.765, \mathrm{P}<0.05)$ in the $\mathrm{PG}$. The total incidence of MACEs in patients with multiple lesions was significantly
\end{abstract}

Correspondence to: Dr Qingmin Wei, Department of Cardiology, Xingtai People's Hospital, 16 Hongxing Street, Qiaodong, Xingtai, Hebei 054000, P.R. China

E-mail: qingminweiyx@163.com

Key words: myeloperoxidase, homocysteine, high-sensitivity C-reactive protein, coronary artery disease, prognosis higher than that in patients with double and single lesions $(\mathrm{P}<0.05)$. The total incidence of MACEs in the MVG group was higher than that in the SVG and the DVG, and the total incidence of MACEs in the DVG was higher than that in the SVG $(\mathrm{P}<0.05)$. The area under the curve (AUC) and sensitivity for MPO levels to predict MACEs were higher than those of Hcy and hs-CRP $(\mathrm{P}<0.05)$; however, there was no significant difference in the AUC and sensitivity of Hcy and hs-CRP for predicting MACEs $(\mathrm{P}<0.05)$. The specificity of hs-CRP for predicting MACEs was higher than that of MPO and Hcy $(\mathrm{P}<0.05)$. The number of lesions, hypertension, diabetes, MPO, Hys and hs-CRP were determined to be independent risk factors for MACEs. In conclusion, for patients with CAD, elevated plasma levels of MPO, Hcy and hs-CRP were directly correlated with the severity of CAD and the risk of MACEs. Furthermore, MPO, Hcy and hs-CRP may effectively predict MACEs and are of important clinical significance in terms of judging the condition and improving the prognosis for patients with CAD.

\section{Introduction}

As a common cardiovascular disease, coronary artery disease (CAD) is mainly caused by coronary artery lesions. In recent years, with the change in social environment and living habits, morbidity and mortality have been increasing $(1,2)$. Coronary atherosclerosis is the pathological basis of CAD. Incomplete or complete occlusion of the coronary artery caused by coronary atherosclerosis is also one of the key factors for the occurrence of CAD (3). CAD, particularly the formation of plaque, is likely to cause major adverse cardiovascular events (MACEs) and the occurrence of MACEs is one of the major causes of death in patients with CAD (4). Therefore, the ability to rapidly judge a patient's condition and predict the occurrence of MACEs is particularly important.

Myeloperoxidase (MPO) is an important inflammatory mediator in the progression of atherosclerotic plaque from stable to unstable stages (5). Homocysteine (Hcy) is an important intermediate metabolite of the protein metabolism pathway and it is formed by modification by enzymes after methionine enters the human body. Numerous studies have indicated that Hcy may be an independent risk factor for 
cardiovascular disease and there is a close association between the condition and thrombosis $(6,7)$. High-sensitivity C-reactive protein (hs-CRP) is involved in the inflammatory reaction. As a highly sensitive inflammatory marker, once the body is in an inflammatory state, levels of hs-CRP rapidly increase within a short period of time (8). It has been indicated that hs-CRP also has an important role in CAD and it may be used as a prognostic marker of cardiovascular disease (9). At present, MPO, Hcy and hs-CRP are used as convenient inflammatory markers in the clinic that may be rapidly detected $(10,11)$. However, few studies have explored the association between these factors and the severity of CAD (12).

Therefore, in the present study, the association between the presence or severity of CAD and the expression of MPO, Hcy or hs-CRP was assessed in order to provide further reference data for judging the condition of patients with CAD and selecting treatment options.

\section{Materials and methods}

Patients. A total of 112 patients (58 males and 54 females) with CAD admitted to Xingtai People's Hospital from March 2014 to July 2016 were included in the patient group (PG). All patients were aged between 57-73 years, with an average age of $66.12 \pm 7.47$ years. In addition, 112 healthy individuals who came to Xingtai People's Hospital from March 2014 to July 2016 for physical examinations were included in the control group (CG). The inclusion criterion was as follows: Patients with CAD that had been confirmed by coronary angiography. The exclusion criteria were as follows: Patients with severe infections or immune diseases, other malignant tumors, severe liver and kidney dysfunction, types of severe heart disease other than CAD and cognitive or communication impairment, as well as those who did not wish to participate in the present study. All patients and their families agreed to participate in the study and signed the informed consent form after understanding the procedure and risks. This study was approved by the Ethics Committee of Xingtai People's Hospital (Xingtai, China).

Laboratory indexes. The patient's fasting venous blood $(5 \mathrm{ml})$ was sampled in the morning after admission. The blood sample was centrifuged at a speed of $1500 \mathrm{x} \mathrm{g}$ for $10 \mathrm{~min}$. After centrifugation, serum and plasma were separated. The level of MPO in the plasma was detected by a colorimetric immunoassay (Raleigh Bioengineering Co., Ltd.). The serum level of Hcy was detected by an enzymatic cycling assay (cat. no. 59400370417; Zhonghan Shengtai Biotechnology Co., Ltd.) on a fully automatic biochemical analyzer (BS-380, Shenzhen Mindray Bio-Medical Electronics Co., Ltd.) and the expression of hs-CRP was detected by immunoturbidimetry on a fully automatic biochemical analyzer (Y70105-1, Zhejiang Erkn Biological Technology Co., Ltd.). The operative procedures were performed strictly in accordance with the manufacturer's protocols.

Outcome measures. i) The plasma levels of MPO, Hcy and hs-CRP were compared between the two groups. ii) According to the arteriography results, the patients were divided into the single-vessel disease group (SVG), double-vessel disease group (DVG) or multi-vessel disease group (MVG). The Gensini scores of the three groups were evaluated according to the Gensini score standard (13). iii) The correlations between the Gensini score and the levels of MPO, Hcy and hs-CRP were analyzed. iv) Patients were followed up by telephone consultations for 6 months and MACEs were recorded and compared. MACEs included myocardial infarction, heart failure and cardiogenic death. v) The predictive values of MPO, Hcy and hs-CRP for MACEs were determined and compared.

Statistical analysis. SPSS 19.0 software (IBM Corp.) was used in the present study for statistical analysis. GraphPad Prism 6 software (GraphPad, Inc.) was used to visualize the data in the present study. The $\chi^{2}$ test was used to assess differences in count data. Values are expressed as the mean \pm standard deviation or $\mathrm{n}(\%)$. The independent-samples t-test was used to compare the data between two groups, and ANOVAs were used to compare data among multiple groups. Tukey's tests were used for post hoc tests. Receiver operating characteristic (ROC) curve analysis was used to assess the predictive performance of MPO, Hys and hs-CRP for MACEs. Pearson's linear correlation analysis was used to determine correlations between the parameters and the Gensini score. The Cox regression model was used to analyze the risk factors of MACE. P $<0.05$ was considered to indicate statistical significance.

\section{Results}

Comparison of general data. There were no differences in sex, age and BMI between the two groups $(\mathrm{P}>0.05)$ and the general data were comparable (Table I).

Plasma levels of MPO, Hcy and hs-CRP in the two groups. The plasma levels of MPO, Hcy and hs-CRP in the PG were $2,671.46 \pm 426.85 \mathrm{pmol} / 1,18.91 \pm 6.75 \mu \mathrm{mol} / 1$ and $4.33 \pm 1.59 \mathrm{mg} / \mathrm{l}$, respectively. The plasma levels of MPO, Hcy and hs-CRP in the $\mathrm{CG}$ were $351.67 \pm 32.89 \mathrm{pmol} / \mathrm{l}, 11.32 \pm 4.12 \mu \mathrm{mol} / \mathrm{l}$ and $1.12 \pm 0.45 \mathrm{mg} / 1$, respectively. Of note, the levels of MPO, Hcy and hs-CRP in the PG were higher than those in the CG $(\mathrm{P}<0.001$; Table II).

Gensini score in the PG. According to their arteriography results, the patients were stratified into the SVG, DVG and MVG groups. The Gensini score of each group was then compared. The Gensini scores of the SVG, DVG and MVG were $12.68 \pm 4.33,16.95 \pm 5.15$ and $22.35 \pm 5.71$, respectively. The Gensini score of the MVG was higher than that of the SVG and the DVG, and the Gensini score of the DVG was higher than that of the SVG $(\mathrm{P}<0.05)$. This indicated that the severity of coronary artery lesions in patients with multi-vessel disease was higher than that in patients with double-vessel and single-vessel disease, and the severity of coronary artery lesions in patients with double-vessel disease was higher than that in patients with single-vessel disease (Fig. 1).

Correlation between Gensini score and MPO, Hcy or $h s-C R P$. The levels of MPO, Hcy and hs-CRP were $2,149.83 \pm 359.61 \mathrm{pmol} / \mathrm{l}, 13.57 \pm 4.26 \mu \mathrm{mol} / 1$ and $3.11 \pm 1.02 \mathrm{mg} / \mathrm{l}$, respectively, in the $\mathrm{SVG}, 2,651.33 \pm 419.65 \mathrm{pmol} / 1$, $18.69 \pm 6.51 \mu \mathrm{mol} / \mathrm{l}$ and $4.39 \pm 1.41 \mathrm{mg} / \mathrm{l}$, respectively, in the 
Table I. Clinicopathological characteristics of the patients and comparison of demographic data between the patient and control groups.

\begin{tabular}{|c|c|c|c|c|}
\hline Item & Patient group $(n=112)$ & Control group $(n=112)$ & $t / \chi^{2}$ & P-value \\
\hline Sex & & & 0.018 & 0.894 \\
\hline Male & $58(51.79)$ & $59(52.68)$ & & \\
\hline Female & $54(48.21)$ & $53(47.32)$ & & \\
\hline Age (years) & & & 0.018 & 0.893 \\
\hline$\geq 66$ & $61(54.46)$ & $60(53.57)$ & & \\
\hline$<66$ & $51(45.53)$ & $52(46.43)$ & & \\
\hline $\mathrm{BMI}\left(\mathrm{kg} / \mathrm{m}^{2}\right)$ & & & 0.018 & 0.894 \\
\hline$\geq 23$ & $57(50.89)$ & $58(51.79)$ & & \\
\hline$<23$ & $55(49.11)$ & $54(48.21)$ & & \\
\hline Number of lesions & & & - & - \\
\hline Single & $41(36.61)$ & - & & \\
\hline Double & $39(34.82)$ & - & & \\
\hline Multiple & $32(28.57)$ & - & & \\
\hline \multicolumn{5}{|c|}{ Coagulation function } \\
\hline APTT $(\mathrm{sec})$ & $28.65 \pm 2.72$ & $29.03 \pm 2.65$ & 1.059 & 0.251 \\
\hline PT (sec) & $12.24 \pm 1.11$ & $12.18 \pm 0.94$ & 0.437 & 0.663 \\
\hline FIB $(g / 1)$ & $3.03 \pm 0.14$ & $3.05 \pm 0.15$ & 1.032 & 0.303 \\
\hline $\mathrm{TT}(\mathrm{sec})$ & $14.65 \pm 1.44$ & $14.53 \pm 1.38$ & 0.637 & 0.525 \\
\hline History of surgery & & & 0.019 & 0.889 \\
\hline Yes & $41(36.61)$ & $40(35.71)$ & & \\
\hline No & $71(63.39)$ & $72(64.29)$ & & \\
\hline \multicolumn{5}{|c|}{ Renal function indices $(\mu \mathrm{mol} / \mathrm{l})$} \\
\hline Creatinine & $63.22 \pm 4.17$ & $63.86 \pm 4.19$ & 1.146 & 0.253 \\
\hline Urea & $5.47 \pm 0.66$ & $5.53 \pm 0.79$ & 0.617 & 0.538 \\
\hline Uric acid & $331.87 \pm 13.64$ & $330.98 \pm 14.05$ & 0.481 & 0.631 \\
\hline
\end{tabular}

BMI, body mass index; FIB, fibrinogen; PT, prothrombin time; APTT, advanced partial thromboplastin time; TT, thrombin time.

Table II. Expression of plasma MPO and serum Hcy and hs-CRP levels in the two groups.

\begin{tabular}{lccr}
\hline Parameter & Patient group $(\mathrm{n}=112)$ & Control group $(\mathrm{n}=112)$ & $\mathrm{t}$ \\
\hline MPO $(\mathrm{pmol} / \mathrm{l})$ & $2,671.46 \pm 426.85$ & $351.67 \pm 32.89$ & 57.35 \\
Hcy $(\mu \mathrm{mol} / \mathrm{l})$ & $18.91 \pm 6.75$ & $11.32 \pm 4.12$ & 10.16 \\
hs-CRP $(\mathrm{mg} / \mathrm{l})$ & $4.33 \pm 1.59$ & $1.12 \pm 0.45$ & 20.56 \\
\hline
\end{tabular}

MPO, myeloperoxidase; Hcy, homocysteine; hs-CRP, high-sensitivity C-reactive protein.

DVG and $3,169.42 \pm 466.73 \mathrm{pmol} / 1,23.88 \pm 7.24 \mu \mathrm{mol} / 1$ and $5.46 \pm 1.63 \mathrm{mg} / \mathrm{l}$, respectively, in the MVG. The levels of MPO, Hcy and hs-CRP in the MVG were higher than those in the DVG and the SVG, and those in the DVG were higher than those in the SVG $(\mathrm{P}<0.05$; Table III). There was a positive correlation between the Gensini score and the levels of MPO (r=0.814, P<0.05; Fig. 2), Hcy ( $r=0.774$, P<0.05; Fig. 3) and hs-CRP ( $r=0.765, \mathrm{P}<0.05$; Fig. 4). This showed that high expressions of plasma MOP, serum Hys and hs-CRP indicated a severe degree of disease progression in patients with CAD.
MACEs in the PG. A total of 37 MACEs were recorded for the 112 patients in the $P G$, including 7 cases of cardiac death and 30 re-hospitalized patients with heart failure or myocardial infarction. Among them, there were 0 cases of cardiac death in the SVG and 7 re-hospitalized patients with heart failure or myocardial infarction, and the total incidence of MACEs was $17.07 \%$. There were 2 cases of cardiac death in the DVG and 10 re-hospitalized patients with heart failure or myocardial infarction, and the total incidence of MACEs was $30.77 \%$. There were 5 cases of cardiac death in the MVG and 
Table III. Plasma levels of MPO, serum Hcy and hs-CRP in patients with different numbers of vessels involved.

\begin{tabular}{lcccr}
\hline Parameter & SVG $(\mathrm{n}=41)$ & DVG $(\mathrm{n}=39)$ & MVG $(\mathrm{n}=32)$ & F \\
\hline MPO $(\mathrm{pmol} / \mathrm{l})$ & $2,149.83 \pm 359.61$ & $2,651.33 \pm 419.65$ & $3,169.42 \pm 466.73$ & 54.91 \\
Hcy $(\mu \mathrm{mol} / \mathrm{l})$ & $13.57 \pm 4.26$ & $18.69 \pm 6.51$ & $23.88 \pm 7.24$ & 26.41 \\
hs-CRP $(\mathrm{mg} / \mathrm{l})$ & $3.11 \pm 1.02$ & $4.39 \pm 1.41$ & $5.46 \pm 1.63$ & 27.54 \\
\hline
\end{tabular}

MPO, myeloperoxidase; Hcy, homocysteine; hs-CRP, high-sensitivity C-reactive protein; SVG, single-vessel disease group; DVG, double-vessel disease group; MVG, multiple-vessel disease group.

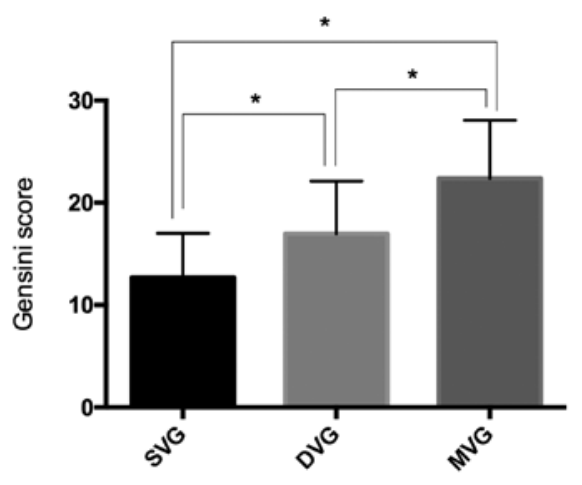

Figure 1. Gensini score for patients in the patient group. The Gensini score of the MVG were higher than those of the SVG and DVG, and the Gensini score of the DVG was higher than that of the SVG $(\mathrm{P}<0.05)$. ${ }^{*} \mathrm{P}<0.05$. SVG, single-vessel disease group; DVG, double-vessel disease group; MVG, multiple-vessel disease group.

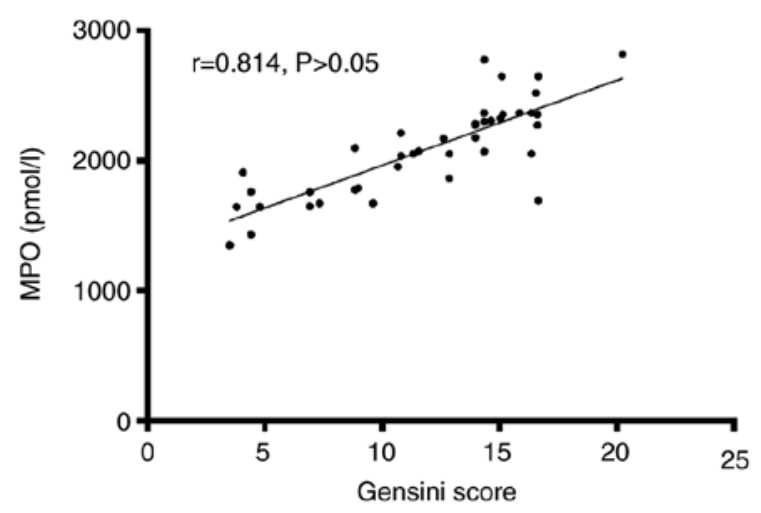

Figure 2. Association between plasma MPO and Gensini score. Correlation between Gensini Score and expression of MPO $(r=0.814, \mathrm{P}>0.05)$. MPO, myeloperoxidase.

13 re-hospitalized patients with heart failure or myocardial infarction, and the total incidence of MACEs was $56.25 \%$. The total incidence of MACEs in the MVG was higher than that in the DVG and SVG $(\mathrm{P}<0.05)$. Furthermore, the total incidence of MACEs in the DVG was higher than that in the SVG $(\mathrm{P}<0.05$; Table IV).

Predictive value of the levels of MPO, Hcy and hs-CRP regarding MACEs. The 112 patients in the PG were divided into the MACE and non-MACE groups based on whether any MACE occurred. The area under the ROC curve (AUC) and prediction sensitivity of MPO regarding MACEs were higher

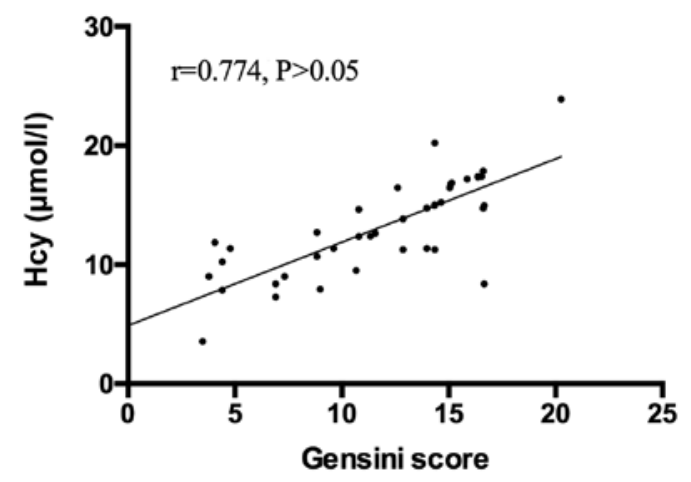

Figure 3. Association between plasma Hcy and Gensini score. Correlation between Gensini Score and expression of Hcy $(r=0.774, \mathrm{P}>0.05)$. Hcy, homocysteine.

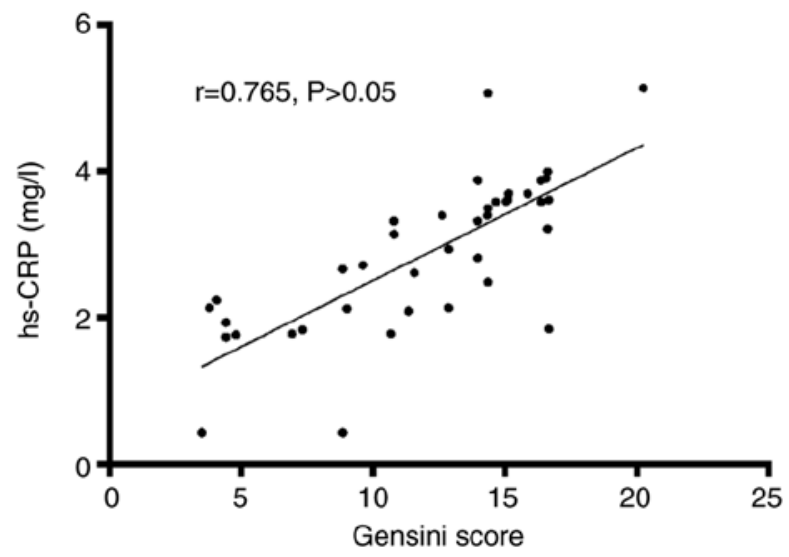

Figure 4. Association between plasma hs-CRP and Gensini score. Correlation between Gensini score and expression of hs-CRP ( $r=0.765, \mathrm{P}>0.05)$. hs-CRP, high-sensitivity C-reactive protein.

than those of Hcy and hs-CRP $(\mathrm{P}<0.05)$. There was no significant difference in AUC and prediction sensitivity between Hcy and hs-CRP $(\mathrm{P}<0.05)$. The specificity of MACE prediction with hs-CRP was higher than that of MPO and Hcy $(\mathrm{P}<0.05$; Table V, Fig. 5).

Univariate analysis of MACEs. Univariate analysis suggested that the number of lesions, hypertension, diabetes, MPO, Hys and hs-CRP were significantly associated with the occurrence of MACEs $(\mathrm{P}<0.05)$. However, sex, age, BMI and smoking habits were not significantly associated with MACE $(\mathrm{P}>0.05$, Table VI). 
Table IV. MACEs in patients with different numbers of vessels involved.

\begin{tabular}{lccrr}
\hline MACE & SVG $(\mathrm{n}=41)$ & DVG $(\mathrm{n}=39)$ & MVG $(\mathrm{n}=32)$ & $\chi^{2}$ \\
\hline Cardiogenic death & $0(0.0)$ & $2(5.13)$ & $5(15.63)$ & 7.617 \\
Heart failure & $3(7.320)$ & $4(10.26)$ & $6(18.75)$ & 0.022 \\
Myocardial infarction & $4(9.76)$ & $6(15.38)$ & $7(21.88)$ & 0.302 \\
Total incidence & $7(17.07)$ & $12(30.77)$ & $18(56.25)$ & 2.052 \\
\hline
\end{tabular}

MACE, major adverse cardiovascular event; SVG, single-vessel disease group; DVG, double-vessel disease group; MVG, multiple-vessel disease group.

Table V. Predictive value of plasma MPO, serum Hcy and hs-CRP expression for major adverse cardiovascular events.

\begin{tabular}{lcccc}
\hline Parameter & Sensitivity $(\%)$ & Specificity $(\%)$ & AUC & Cut-off value \\
\hline MPO (pmol/l) & 78.67 & $56.76^{\mathrm{a}}$ & 0.812 & 2,906 \\
Hcy $(\mu \mathrm{mol} / \mathrm{l})$ & $73.33^{\mathrm{b}}$ & $54.05^{\mathrm{a}}$ & $0.711^{\mathrm{b}}$ & 20.30 \\
hs-CRP $(\mathrm{mg} / \mathrm{l})$ & $69.33^{\mathrm{b}}$ & 62.16 & $0.715^{\mathrm{b}}$ & 4.795 \\
\hline
\end{tabular}

${ }^{\mathrm{a}} \mathrm{P}<0.05$ vs. hs-CRP; ${ }^{\mathrm{b}} \mathrm{P}<0.05$ vs. MPO. MPO, myeloperoxidase; Hcy, homocysteine; hs-CRP, high-sensitivity C-reactive protein; AUC, area under curve.

Table VI. Univariate analysis for the association of various parameters with MACEs.

\begin{tabular}{|c|c|c|c|c|}
\hline Item & MACE group $(n=37)$ & Non-MACE group $(n=75)$ & $\chi^{2}$ & P-value \\
\hline Sex & & & 0.114 & 0.736 \\
\hline Male $(n=58)$ & $20(54.05)$ & $38(50.67)$ & & \\
\hline Female $(n=54)$ & $17(45.95)$ & $37(49.33)$ & & \\
\hline Age (years) & & & 0.117 & 0.732 \\
\hline$\geq 66(n=61)$ & $21(56.76)$ & $40(53.33)$ & & \\
\hline$<66(\mathrm{n}=51)$ & $16(43.24)$ & $35(46.67)$ & & \\
\hline BMI $\left(\mathrm{kg} / \mathrm{m}^{2}\right)$ & & & 0.005 & 0.946 \\
\hline$\geq 23(\mathrm{n}=57)$ & $19(51.35)$ & $38(50.67)$ & & \\
\hline$<23(\mathrm{n}=55)$ & $18(48.65)$ & $37(49.33)$ & & \\
\hline Number of lesions & & & 18.24 & $<0.001$ \\
\hline Single $(n=41)$ & $7(18.92)$ & $34(45.33)$ & & \\
\hline Double $(n=39)$ & $10(27.03)$ & $29(38.67)$ & & \\
\hline Multiple $(n=32)$ & $20(54.05)$ & $12(16.00)$ & & \\
\hline Hypertension & & & 22.66 & $<0.001$ \\
\hline Yes & $31(83.78)$ & $27(36.00)$ & & \\
\hline No & $6(16.22)$ & $48(64.00)$ & & \\
\hline Diabetes & & & 20.14 & $<0.001$ \\
\hline Yes & $29(73.38)$ & $25(33.33)$ & & \\
\hline No & $8(21.62)$ & $50(66.67)$ & & \\
\hline Smoking & & & 0.040 & 0.841 \\
\hline Yes & $18(48.65)$ & $38(50.67)$ & & \\
\hline No & $19(51.35)$ & 37 (49.33) & & \\
\hline MPO (pmol/l) & 2,998.331/1))74 & $2,465.91 \pm 462.55$ & 6.133 & $<0.001$ \\
\hline Hcy $(\mu \mathrm{mol} / \mathrm{l})$ & $20.51 \pm 5.43$ & $16.59 \pm 6.21$ & 3.217 & $<0.001$ \\
\hline hs-CRP (mg/l) & $5.15 \pm 1.59$ & $3.97 \pm 1.59$ & 3.694 & $<0.001$ \\
\hline
\end{tabular}

BMI, body mass index; MPO, myeloperoxidase; Hcy, homocysteine; hs-CRP, high-sensitivity C-reactive protein; MACE, major adverse cardiovascular event. 
Table VII. Assignments.

Factor

Number of lesions

Hypertension

Diabetes

MPO

Hys

hs-CRP
Assignment

Single and double; 1 ; mutiple, 2

Yes, 1 ; No, 2

Yes, 1 ; No, 2

Continuous variable and analyzed using raw data

Continuous variable and analyzed using raw data

Continuous variable and analyzed using raw data

MPO, myeloperoxidase; Hcy, homocysteine; hs-CRP, high-sensitivity C-reactive protein.

Table VIII. Multivariate analysis of the association of various factors on major adverse cardiovascular events.

\begin{tabular}{lccccc}
\hline Factor & $\beta$ & SE & Wald & HR & 95\% CI \\
\hline Number of lesions & 0.346 & 0.155 & 5.448 & 1.452 & $1.019-1.885$ \\
Hypertension & 1.154 & 0.519 & 4.784 & 3.206 & $1.121-9.163$ \\
Diabetes & 1.004 & 0.321 & 9.409 & 2.743 & $1.441-5.222$ \\
MPO & 0.564 & 0.118 & 9.482 & 2.679 & $1.734-4.319$ \\
Hys & 0.502 & 0.088 & 8.624 & 2.427 & $1.506-3.773$ \\
hs-CRP & 0.422 & 0.131 & 6.255 & 1.751 & $1.168-2.355$ \\
\hline
\end{tabular}

MPO, myeloperoxidase; Hcy, homocysteine; hs-CRP, high-sensitivity C-reactive protein; SE, standard error; HR, hazard ratio.
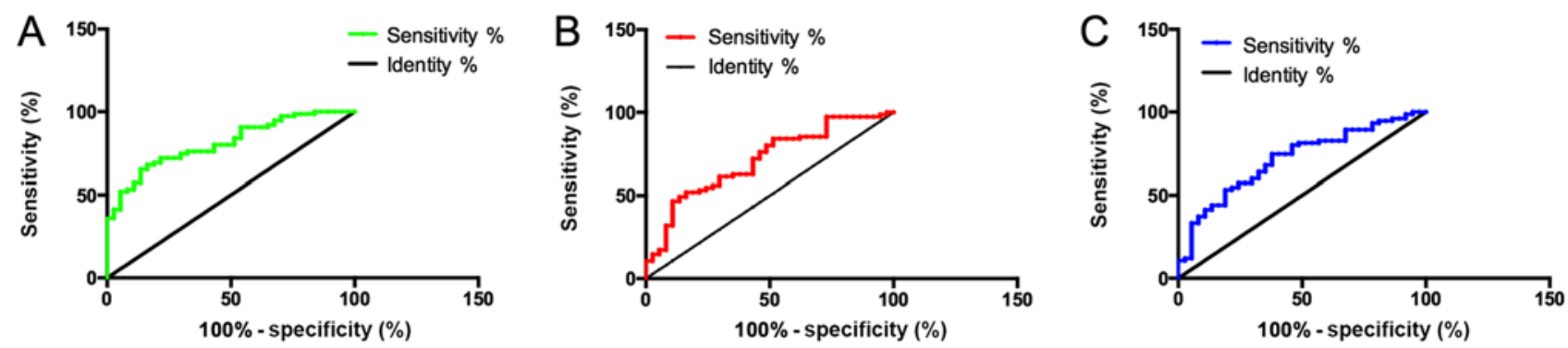

Figure 5. Receiver operating characteristic curve analysis for determining the predictive value of plasma MPO, Hcy and hs-CRP for MACE. (A) Predictive value of expression of MPO for MACE. The sensitivity, specificity and AUC of plasma MPO for predicting MACE were 78.67\%, 56.76\% and 0.812 , respectively. The cut-off value was $2906 \mathrm{pmol} / 1$. (B) Predictive value of expression of Hcy for MACE. The sensitivity, specificity and AUC of plasma MPO for predicting MACE were 73.33\%, 54.05\% and 0.711, respectively. The cut-off value was $20.30 \mu \mathrm{mol} / 1$. (C) Predictive value of expression of hs-CRP for MACE. The sensitivity, specificity and AUC of plasma MPO for predicting MACE were $69.33 \%, 62.16 \%$ and 0.715 , respectively. The cut-off value was $4.795 \mathrm{mg} / 1$. MPO, myeloperoxidase; Hcy, homocysteine; hs-CRP, high-sensitivity C-reactive protein; AUC, area under curve; MACE, major adverse cardiovascular event.

Multivariate analysis of MACEs. The number of lesions, hypertension, diabetes, MPO, Hys and hs-CRP were taken as independent variables and they were assigned with values (Table VII). The occurrence of MACEs was treated as a dependent variable and logistic regression analysis was adopted for multivariate analysis. It was revealed that the number of lesions, hypertension, diabetes, MPO, Hys and hs-CRP were independent risk factors for MACEs (Table VIII).

\section{Discussion}

With the incidence of $\mathrm{CAD}$ on the rise, the condition is increasingly threatening human life and health (14).
If patients are not treated in a timely manner, they may experience MACEs including cardiogenic death, and their survival and safety may be seriously affected (15). Studies have indicated that CAD is caused by a variety of factors and certain biochemical indicators have been clinically proven to be closely linked to CAD (16). For instance, MPO, as a lysosomal enzyme in the cytoplasm of neutrophilic granulocytes, is a potential mediator of vascular injury. The oxidative products of MPO released by granulocytes during an inflammatory reaction are closely associated with the occurrence of atherosclerosis $(17,18)$. Hcy and hs-CRP are also biochemical indicators that are considered to be closely linked to CAD $(19,20)$. In the present study, the value of 
MPO, Hcy and hs-CRP to predict the severity and of CAD and the prognosis of affected patients was assessed with the aim of providing more accurate biochemical data for the evaluation of CAD and selection of treatments.

The present results indicated that the plasma levels of MPO, Hcy and hs-CRP in the PG were higher than those in CG. A previous study indicated high expression of MPO in venous blood of patients with acute coronary syndrome (21). Another study demonstrated that MPO may serve as an inflammatory marker and that it damages vascular endothelium by oxidizing cholesterol and low-density lipoprotein while degrading the extracellular matrix and corroding patches (22). Furthermore, another study has indicated that hs-CRP and Hcy levels were significantly increased in patients with CAD and that the expression of hs-CRP and Hcy varied with the severity of CAD (23). The results of these studies were consistent with those of the present study. Patients in the PG were divided into three groups, SVG, DVG and MVG, according to the arteriography results. The Gensini scores of the three groups were evaluated and the correlations between the plasma levels of MPO, Hcy or hs-CRP and the Gensini score were determined. The results indicated that the Gensini score and expression of MPO, Hcy and hs-CRP in the MVG were higher than those in the DVG and SVG, and the Gensini score and expression of MPO, Hcy and hs-CRP in the DVG were higher than those in the SVG $(\mathrm{P}<0.05)$. There was a positive correlation between the Gensini score and the levels of MPO, Hcy and hs-CRP, which indicated that higher expression of MPO, Hcy and hs-CRP were directly linked to the severity of the CAD. Next, the MACEs recorded were compared among the three groups with different degrees of lesions and the results suggested that the total incidence of MACEs in the MVG was higher than that in the SVG and the DVG, and the total incidence of MACEs in the DVG was higher than that in the SVG $(\mathrm{P}<0.05)$. This further indicated that higher expression of MPO, Hcy and hs-CRP was directly linked to the severity of CAD and increased risk of MACEs. A previous study explored the association between MPO and acute coronary syndrome, indicating that the concentration of MPO in the plasma of patients with acute coronary syndrome is able to predict the risk of heart disease (24); higher levels of MPO were associated with a higher risk of death, and therefore, this was consistent with the conclusion of the present study. In terms of the expression of Hcy and hs-CRP in the serum, one study suggested that high levels of Hcy are able to cause damage to blood vessels and destroy the elasticity of the blood vessel wall (25). One study demonstrated a positive correlation between the increased expression of Hcy and the incidence of acute coronary syndrome and MACEs (26). Hs-CRP may induce macrophages to absorb and oxidize low-density lipoproteins, and it is also able to stimulate macrophages to release pre-thrombotic tissue factor (27). In addition, one study suggested that the expression of hs-CRP varied in the sera of patients with different degrees of CAD (28). The severity of CAD was directly linked to the expression of hs-CRP (29). All of the above studies confirmed the conclusions of the present study and briefly explained the mechanism. Finally, the predictive value of MPO, Hcy and hs-CRP for MACEs was analyzed. The results suggested that the three factors had high predictive value for MACEs and the predictive value of MPO for MACEs was higher than that of the other two factors. This suggested that MPO, Hcy and hs-CRP are somewhat accurate in predicting MACE, but the predictive value of MPO is higher, although hs-CRP showed a higher specificity. A previous study explored the predictive value of MPO and Hcy for MACEs in patients with acute coronary syndrome and demonstrated that MPO and Hcy had a high predictive value (30). A further study clearly indicated that MPO has a higher predictive value for MACEs in patients with CAD within six months when compared to other conventional indicators (31). This was similar to the result of the present study. Logistic multivariate analysis indicated that the number of lesions, hypertension, diabetes, MPO, Hys and hs-CRP were independent risk factors for MACEs, suggesting that the prognosis of patients may be improved by prompt intervention in these factors. A previous study has clearly indicated that MPO and hs-CRP may be used to predict MACEs in patients with CAD (32). In addition, the combination of hs-CRP and Hcy had a high predictive value for increased risk of coronary artery disease in a Korean population (33). This is consistent with the present results.

In conclusion, elevated plasma levels of MPO, Hcy and hs-CRP were directly associated with the severity of CAD and the risk of MACEs. MPO, Hcy and hs-CRP may be used to effectively predict the occurrence of MACEs in patients with CAD, and they have important clinical significance for judging the condition of patients and improving prognosis. However, the present study has certain limitations. For instance, the specific mechanism underlying the role of MPO, Hcy and hs-CRP in CAD remains to be fully explored and only relevant studies were cited to briefly describe the mechanism. In future studies, the association of MPO, Hcy and hs-CRP with CAD, as well as the mechanism, should be further verified.

\section{Acknowledgements}

Not applicable.

\section{Funding}

No funding was received.

\section{Availability of data and materials}

The datasets used and/or analyzed during the current study are available from the corresponding author on reasonable request.

\section{Authors' contributions}

$\mathrm{MuC}$ and $\mathrm{MgC}$ conceived and designed the study and interpreted the results. QW contributed to the design of the study and interpretation of the results. $\mathrm{MuC}$ and $\mathrm{QW}$ performed experiments, analyzed data, prepared figures and drafted the manuscript. $\mathrm{MuC}, \mathrm{MgC}$ and $\mathrm{QW}$ approved final version of the manuscript. $\mathrm{mgC}$ edited and revised the manuscript. All authors read and approved the final manuscript. 


\section{Ethics approval and consent to participate}

This study was approved by the Ethics Committee of Xingtai People's Hospital (Xingtai, China). All study participants provided written informed consent prior to participating in the study.

\section{Patient consent for publication}

Not applicable.

\section{Competing interests}

The authors declare that they have no competing interests.

\section{References}

1. Ahmadi A, Min J, Berger A, Achenbach S, Alaamri S, Al-Mallah M, Berman D, Budoff M, Cademartiri F, Callister T, et al: Does prognosis change depending on number and composition of non obstructive plaques? Results from the multinational coronary CT angiography evaluation for clinical outcome: An international multicenter registry (CONFIRM). J Am Coll Cardiol 63: A980, 2014.

2. Holman RR, Coleman RL, Chan JCN, Chiasson J-L, Feng H, Ge J, Gerstein HC, Gray R, Huo Y, Lang Z, et al; ACE Study Group: Effects of acarbose on cardiovascular and diabetes outcomes in patients with coronary heart disease and impaired glucose tolerance (ACE): A randomised, double-blind, placebo-controlled trial. Lancet Diabetes Endocrinol 5: 877-886, 2017.

3. Li H, Sun K, Zhao R, Hu J, Hao Z, Wang F, Lu Y, Liu F and Zhang Y: Inflammatory biomarkers of coronary heart disease. Front Biosci 22: 504-515, 2017.

4. Brown AJ, Shah ASV, West NEJ, Costopoulos C, Orzalkiewicz M, Newby DE, Bennett MR, Mills NL and Calvert PA: High-Sensitivity Troponin I Is Associated With High-Risk Plaque and MACE in Stable Coronary Artery Disease. JACC Cardiovasc Imaging 10A: A1200-A1203, 2017.

5. Loria V, Dato I, Graziani F and Biasucci LM: Myeloperoxidase: A new biomarker of inflammation in ischemic heart disease and acute coronary syndromes. Mediators Inflamm 2008: $135625-135625,2008$

6. Masud R and Baqai HZ: The communal relation of MTHFR, MTR, ACE gene polymorphisms and hyperhomocysteinemia as conceivable risk of coronary artery disease. Appl Physiol Nutr Metab 42: 1009-1014, 2017

7. Karolczak K, Kubalczyk P, Glowacki R, Pietruszynski R and Watala C: Aldosterone modulates blood homocysteine and cholesterol in coronary artery disease patients - a possible impact on atherothrombosis? Physiol Res 67: 197-207, 2018

8. Tayefi M, Tajfard M, Saffar S, Hanachi P, Amirabadizadeh AR, Esmaeily H, Taghipour A, Ferns GA, Moohebati M and Ghayour-Mobarhan M: hs-CRP is strongly associated with coronary heart disease (CHD): A data mining approach using decision tree algorithm. Comput Methods Programs Biomed 141: 105-109, 2017.

9. Nortamo S, Ukkola O, Lepojärvi S, Kenttä T, Kiviniemi A, Junttila J, Huikuri H and Perkiömäki J: Association of sST2 and hs-CRP levels with new-onset atrial fibrillation in coronary artery disease. Int J Cardiol 248: 173-178, 2017.

10. Rifaie O, Ahmed Abdel-Rahman M and Mabrouk Salem Omar A: Favorable inflammatory status following intermittent fasting in patients with atherosclerotic coronary heart disease after revascuralization. Eur Heart J 34 (Suppl 1): P5502, 2013

11. Yang K, Dong G, Tian Y and Li J: Effects of compound Danshen injection combined with magnesium sulfate on serum MPO and hs-CRP in patients with severe preeclampsia. Exp Ther Med 16: 167-170, 2018.

12. Liu C, Chen L, Yang Y, Huang C, Luo J and Peng D: Myeloperoxidase and high-sensitivity C-reactive protein for predicting major adverse cardiovascular events in patients with coronary heart disease. Int J Clin Med 6: 262-270, 2015.
13. Nurkalem Z, Hasdemir H, Ergelen M, Aksu H, Sahin I, Erer B, Yilmaz HY, Comert N, Sargin M and Eren M: The relationship between glucose tolerance and severity of coronary artery disease using the Gensini score. Angiology 61: 751-755, 2010.

14. Zhao W, Rasheed A, Tikkanen E, Lee J-J, Butterworth AS, Howson JMM, Assimes TL, Chowdhury R, Orho-Melander M, Damrauer S, et al; CHD Exome+ Consortium; EPIC-CVD Consortium; EPIC-Interact Consortium; Michigan Biobank: Identification of new susceptibility loci for type 2 diabetes and shared etiological pathways with coronary heart disease. Nat Genet 49: 1450-1457, 2017.

15. Lee JH, Han D, Hartaigh BÓ, Gransar H, Lu Y, Rizvi A, Park MW, Roudsari HM, Stuijfzand WJ, Berman DS, et al: Influence of symptom typicality for predicting MACE in patients without obstructive coronary artery disease: From the CONFIRM Registry (Coronary Computed Tomography Angiography Evaluation for Clinical Outcomes: An International Multicenter Registry). Clin Cardiol 41: 586-593, 2018.

16. Hashemian M, Poustchi H, Mohammadi-Nasrabadi F and Hekmatdoost A: Systematic review of zinc biochemical indicators and risk of coronary heart disease. ARYA Atheroscler 11: 357-365, 2015.

17. Luyao C, Shushan Z, Ruizheng S and Guogang Z: GW24-e0526 The Relationship Between MPO-463 G/A, MPO-129 G/A Gene Polymorphism and Coronary Heart Disease: A Meta-Analysis. Heart 99 (Suppl 3): A117-A118, 2013.

18. Hu J, Xu J, Zhou X, Jiang T, Li Y and Zhang M: Correlation between MPO $129 \mathrm{~A} / \mathrm{G}$ polymorphism and severity of coronary artery disease. Zhongguo Ying Yong Sheng Li Xue Za Zhi 27: 306-310, 2011

19. Bjørnestad EØ, Borsholm RA, Svingen GFT, Pedersen ER, Seifert R, Midttun Ø, Ueland PM, Tell GS, Bønaa KH and Nygård O: Neopterin as an Effect Modifier of the Cardiovascular Risk Predicted by Total Homocysteine: A Prospective 2-Cohort Study. J Am Heart Assoc 6: e006500, 2017.

20. Wang L-Y, Zhang H-L and Chen S: Effect of atorvastatin combined with trimetazidine on oxidative stress, hemorheology and NT-proBNP, hs-CRP in patients with coronary heart disease. Hainan Yixueyuan Xuebao 23: 2323-2327, 2017.

21. Fong SW, Few LL, See Too WC, Khoo BY, Nik Ibrahim NN, Yahaya SA, Yusof Z, Mohd Ali R, Abdul Rahman AR and Yvonne-Tee GB: Systemic and coronary levels of CRP, MPO, sCD40L and PlGF in patients with coronary artery disease. BMC Res Notes 8: 679, 2015.

22. Karakas M, Koenig W, Zierer A, Herder C, Rottbauer W, Baumert J, Meisinger C and Thorand B: Myeloperoxidase is associated with incident coronary heart disease independently of traditional risk factors: Results from the MONICA/KORA Augsburg study. J Intern Med 271: 43-50, 2012.

23. Mansori K and Ayubi E: Comments on association of sST2 and hs-CRP levels with new-onset atrial fibrillation in coronary artery disease. Int J Cardiol 257: 32, 2018.

24. Zhang X, Dong L, Wang Q and Xie X: The relationship between fasting plasma glucose and MPO in patients with acute coronary syndrome. BMC Cardiovasc Disord 15: 93, 2015.

25. Huang X, Lv X, Song H, Yang Q, Sun Y, Zhang W, Yu X, Dong $\mathrm{S}$, Yao $\mathrm{W}$, Li Y, et al: The relationship between S-adenosylhomocysteine and coronary artery lesions: A case control study. Clin Chim Acta 471: 314-320, 2017.

26. Szalai AJ, McCrory MA, Xing D, Hage FG, Miller A, Oparil S, Chen Y-F, Mazzone M, Early R, Henry SP, et al: Inhibiting C-reactive protein for the treatment of cardiovascular disease: promising evidence from rodent models. Mediators Inflamm 2014: 353614, 2014.

27. Li S, Jiao Y, Wang H, Shang Q, Lu F, Huang L, Liu J, Xu H and Chen K: Sodium tanshinone IIA sulfate adjunct therapy reduces high-sensitivity $\mathrm{C}$-reactive protein level in coronary artery disease patients: A randomized controlled trial. Sci Rep 7: 17451, 2017.

28. Abhashi SA, Kryeziu FU and Nazreku FD: Increased carotid intima-media thickness associated with high hs-CRP levels is a predictor of unstable coronary artery disease. Cardiovasc J Afr 24: 270-273, 2013.

29. Tajfard M, Tavakoly Sany SB, Avan A, Latiff LA, Rahimi HR, Moohebati M, Hasanzadeh M, Ghazizadeh H, Esmaeily H, Doosti $\mathrm{H}$, et al: Relationship between serum high sensitivity C-reactive protein with angiographic severity of coronary artery disease and traditional cardiovascular risk factors. J Cell Physiol 234: 10289-10299, 2019. 
30. Emami Razavi A, Basati G, Varshosaz J and Abdi S: Association between HDL particles size and myeloperoxidase/ paraoxonase-1 (MPO/PON1) ratio in patients with acute coronary syndrome. Acta Med Iran 51: 365-371, 2013.

31. Kaya mg, Yalcin R, Okyay K, Poyraz F, Bayraktar N, Pasaoglu H, Boyaci B and Cengel A: Potential role of plasma myeloperoxidase level in predicting long-term outcome of acute myocardial infarction. Tex Heart Inst J 39: 500-506, 2012.

32. Tang WH, Shrestha K, Troughton RW, Borowski AG and Klein AL: Integrating plasma high-sensitivity $\mathrm{C}$-reactive protein and myeloperoxidase for risk prediction in chronic systolic heart failure. Congest Heart Fail 17: 105-109, 2011.
33. Cho D-Y, Kim K-N, Kim K-M, Lee D-J and Kim B-T: Combination of high-sensitivity $\mathrm{C}$-reactive protein and homocysteine may predict an increased risk of coronary artery disease in Korean population. Chin Med J (Engl) 125: 569-573, 2012.

This work is licensed under a Creative Commons Attribution-NonCommercial-NoDerivatives 4.0 International (CC BY-NC-ND 4.0) License. 